\title{
Outage performance of fixed-gain and variable-gain AF full-duplex relaying in non-identical Nakagami- $m$ fading channels
}

\author{
Asil Koç ${ }^{1}$ ibrahim Altunbaş ${ }^{1}$ and Abbas Yongaçoğlu²* (D)
}

\begin{abstract}
We investigate outage performance of fixed-gain and variable-gain amplify-and-forward (AF) full-duplex (FD) relaying systems under residual loop-interference (LI) in non-identical Nakagami-m fading channels. New exact outage probability expressions are derived analytically in a single-integral form by using the cumulative distribution function approach. Moreover, tight lower-bound and asymptotic outage probability expressions are derived in closed-form for the evaluated systems. It is shown that the fixed-gain AF-FD relaying outperforms the variable-gain AF-FD relaying when $\mathrm{LI}$ power and/or signal-to-noise ratio increase. The analytical results are verified by Monte-Carlo simulations.
\end{abstract}

Keywords: Full-duplex relaying, Nakagami-m, Amplify and forward, Fixed-gain, Variable-gain, Outage analysis

\section{Introduction}

Full-Duplex (FD) communication allows receiving and transmitting the signal at the same time interval using the same frequency band, hence it improves the spectral efficiency and reduces end-to-end delay with respect to the conventional half-duplex communication [1]. Additionally, the FD communication improves the network secrecy by means of the mixed signals during the simultaneous transmission [1]. Therefore, it has recently gained great attention as a potential candidate for the next generation wireless communication systems [2]. Although FD communication causes loop-interference (LI) (or selfinterference) at the receiver antenna, the recent developments on antenna technology and signal processing techniques make it feasible by applying LI cancellation techniques, e.g., antenna separation, analog cancellation and digital cancellation [3-5]. The practical implementations in [4] shows that although the LI power can be decreased $74 \mathrm{~dB}$ on average, it cannot be completely removed, even with complex cancellation processes [4-6]. Moreover, the experimental studies as in [4] demonstrate that it is convenient to model the residual LI channel with

*Correspondence: yongac@uottawa.ca

${ }^{2}$ School of Electrical Engineering and Computer Science, University of Ottawa, Ottawa, Canada

Full list of author information is available at the end of the article
Rician or Nakagami- $m$ fading due to the multipath signal propagation and line-of-sight (LOS) component between the transmitter and the receiver.

Cooperative communication with relaying has also attracted considerable attention in recent years since it provides coverage extension, capacity, outage, and error performance enhancement [7]. Amplify-and-forward (AF) and decode-and-forward (DF) are the most popular signal forwarding methods used at the relays [7]. In the AF method, the amplifying process may be based on the partial or full channel state information of the sourcerelay channel, namely fixed-gain [8] and variable-gain [9] AF relaying, respectively. The conventional cooperative communication systems suffer from spectral efficiency loss due to half-duplex relaying. In order to improve the spectral efficiency, FD communication is utilized at the relays [10]. In the literature, several papers investigate performance of the AF-FD relaying. In [11], optimal power allocation (PA) problem and capacity of the variable-gain AF-FD relaying are investigated. In [12], outage probability of the variable-gain AF-FD relaying system with direct link between source and destination is investigated in Rayleigh fading channels and shown that the direct link provides diversity gain. The outage probability of the variable-gain AF-FD relaying system with direct link is derived for Rayleigh fading channels in [13] under processing delay and residual LI. Note that the source-relay 
link is assumed fading-free in [13]. In [14], optimal PA problem based on statistical and instantaneous channel state information for the variable-gain AF-FD relaying system with direct link is investigated for Rayleigh fading under total source and relay power constraint. In that paper, minimum outage probability expressions are derived and comparisons with the DF-FD relaying are also presented. An optimal PA analysis based on minimizing the pairwise error probability is carried out for the fixedgain AF-FD relaying in [15] and shown that unequal PA presents better error performance than those using equal power in the transmitters. In [16], error performance and diversity of uncoded and coded fixed-gain AF-FD relaying with direct link are investigated. AF-FD relaying is applied to the systems with multiple relays (e.g. in [17-19]) and shown that the performance can be enhanced significantly by using all the relays or the selected relay. The outage performance of variable-gain AF-FD relaying over Nakagami$m$ channel is recently analyzed in [20] and the exact outage probability expression is derived in terms of extended generalized bivariate Meijer-G function (EGBMGF). In [20], an approximate outage performance expression is also obtained in closed-form by ignoring additive noise in the source-relay channel. Besides, the asymptotic outage probability, asymptotic ergodic capacity and ergodic capacity lower-bound expressions are presented. In [21], the outage performance of the fixed-gain AF-FD relaying is investigated and compared with the variable-gain AF-FD relaying over Rayleigh fading channels where the exact outage probability expression is obtained in a singleintegral.

In this paper, we derive exact outage probability expressions for both fixed-gain and variable-gain dualhop AF-FD relaying where all channels are modeled as Nakagami- $m$ fading channel including the residual LI channel. The outage probability expressions are obtained by using cumulative distribution function (CDF) of the end-to-end signal-to-interference-plus-noise ratio (SINR). Our results in the fixed-gain and variable-gain AF-FD relaying are both in a single-integral form. To the best of our knowledge, there is no outage performance investigation in the literature for the fixed-gain AF-FD relaying systems over non-identical Nakagami$m$ fading channels. Also, our exact outage performance result for the variable-gain AF-FD relaying can be easily calculated numerically in comparison to the exact outage probability expression in terms of EGBMGF given in [20] which is based on double Mellin-Barnes type integrals. Furthermore, there is no restriction on the $m$ parameter of the relay-destination link in our analyses. We also present tight lower-bound and asymptotic outage probability expressions of the considered systems in closedform. The theoretical results are validated by computer simulations.
The main contributions of our paper can be summarized as follows:

- All of the channels are modeled as Nakagami-m fading channels including the residual LI channel at the relay node which makes the analyses more accurate for practical purposes.

- The exact outage probability expressions of the fixed-gain AF-FD and variable-gain AF-FD systems are derived in single-integral forms which can be easily evaluated by using common mathematical package programs.

- For both of the considered systems, the exact outage performance analyses are supported with the lower-bound and asymptotic outage probability expressions obtained in closed-form.

- The advantages of the fixed-gain AF-FD relaying with respect to the variable-gain AF-FD relaying in terms of outage performance are presented. We demonstrate that the system has lower error floor when the fixed-gain AF-FD relaying is deployed.

- It is shown that $m$ parameter of the residual LI channel has dominant effect for the performance limitation in the fixed-gain AF-FD systems. On the other hand, the dominant $m$ parameter of the variable-gain AF-FD systems belongs to the source-relay link channel for the error floor.

The remainder of the paper is organized as follows. In Section 2, the system model for AF-FD relaying is introduced and the SINR expressions are derived for the fixed-gain and variable-gain relays. The exact outage probability expressions of the proposed systems are given in Section 3. Then, the lower-bound and asymptotic outage probability analyses are given in Section 4 . The theoretical and simulation results for different scenarios are demonstrated in Section 5. Finally, Section 6 concludes the paper.

\section{System model and end-to-end SINR}

We consider a dual-hop network as in Fig. 1 where the source (S) and the destination (D) both have single antenna. Source and destination communicate with each other through an AF-FD relay (R) and there is no direct link between the source and destination. The relay is equipped with one receive and one transmit antenna. In the figure, $h_{S R}$ and $h_{R D}$ are complex channel coefficients between S-R and R-D links, respectively and $h_{L I}$ is the complex residual LI term. Their envelopes $\left(\left|h_{S R}\right|,\left|h_{R D}\right|,\left|h_{L I}\right|\right)$ are assumed to be independent and non-identically Nakagami- $m$ distributed. When the unitpower signal $x(t)$ is transmitted from the source, the signal received at the receive antenna of the relay is expressed as

$$
r(t)=h_{S R} \sqrt{P_{S}} x(t)+h_{L I} \sqrt{P_{R}} s(t)+n_{R}(t)
$$




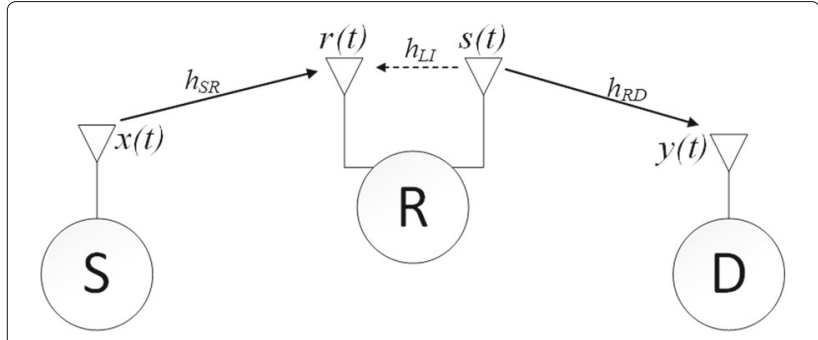

Fig. 1 System model

where $s(t)=\beta r(t-\tau)$ is the signal transmitted by the relay at the same time. $P_{S}$ and $P_{R}$ are the transmission powers at the source and relay, respectively and $n_{R}(t)$ is the complex noise with zero mean and variance $\sigma_{R}^{2}$. $\beta$ is the amplification factor and $\tau$ is the processing delay at the relay. By recursively using (1), the transmitted signal $s(t)$ can be rewritten as

$$
s(t)=\beta \sum_{j=1}^{\infty}\left(\beta h_{L I} \sqrt{P_{R}}\right)^{j-1} g(t-j \tau)
$$

where $g(t)=h_{S R} \sqrt{P_{S}} x(t)+n_{R}(t)$. Finally, the received signal at the destination can be given by $y(t)=$ $h_{R D} \sqrt{P_{R}} S(t)+n_{D}(t)$ where $n_{D}(t)$ is the complex noise with zero mean and variance $\sigma_{D}^{2}$. By analyzing (2), one can easily show that stability of the relay is guaranteed and oscillations are prevented, if the $\beta^{2}<1 /\left(P_{R}\left|h_{L I}\right|^{2}\right)$ condition is satisfied [3]. In that case, the instantaneous transmission power at the relay and the instantaneous end-to-end signal-to-interference-plus-noise ratio (SINR) at the destination are, respectively, given as [3]

$$
\begin{aligned}
& \left\langle P_{R}|s(t)|^{2}\right\rangle=P_{R} \frac{\gamma_{S R}+1}{\frac{1}{\beta^{2} \sigma_{R}^{2}}-\gamma_{L I}}, \\
& \gamma_{\text {end }}=\frac{\gamma_{S R} \gamma_{R D}}{\frac{\gamma_{R D} \gamma_{L I}\left(\gamma_{S R}+1\right)}{\frac{1}{\beta^{2} \sigma_{R}^{2}}-\gamma_{L I}}+\gamma_{R D}+\frac{1}{\beta^{2} \sigma_{R}^{2}}}
\end{aligned}
$$

where $\langle$.$\rangle represents the time average over one symbol$ duration, $\gamma_{S R}=P_{S}\left|h_{S R}\right|^{2} / \sigma_{R}^{2}, \gamma_{R D}=P_{R}\left|h_{R D}\right|^{2} / \sigma_{D}^{2}$ and $\gamma_{L I}=P_{R}\left|h_{L I}\right|^{2} / \sigma_{R}^{2}$. By defining $\bar{\gamma}_{S R}=P_{S} \Omega_{S R} / \sigma_{R}^{2}, \bar{\gamma}_{R D}=$ $P_{R} \Omega_{R D} / \sigma_{D}^{2}, \bar{\gamma}_{L I}=P_{R} \Omega_{L I} / \sigma_{R}^{2}$ and $\Omega_{A}=E\left[\left|h_{A}\right|^{2}\right]$ with $A \in\{S R, R D, L I\}$, probability density function (PDF) and $\mathrm{CDF}$ of these random variables can be given, respectively, by $p_{\gamma_{A}}(x)=\frac{\mu_{A}^{m_{A}} x^{m_{A}-1}}{\Gamma\left(m_{A}\right)} e^{-x \mu_{A}}$ and $F_{\gamma_{A}}(x)=\frac{\gamma\left(m_{A}, x \mu_{A}\right)}{\Gamma\left(m_{A}\right)}$ with $\mu_{A}=m_{A} / \bar{\gamma}_{A}$. Here, $\Gamma$ (.) and $\gamma(.$, .) are the Gamma ([22] eq.(8.310.1)) and the lower incomplete Gamma ([22] eq.(8.350.1)) functions.

\subsection{Fixed-gain relaying}

By choosing the amplification factor $\beta=1 / \sqrt{P_{S} \Omega_{S R}+P_{R} \Omega_{L I}+\sigma_{R}^{2}}$, the instantaneous SINR is obtained as [21]

$$
\gamma_{\text {end }}=\left\{\begin{array}{cc}
\frac{\gamma_{S R} \gamma_{R D}\left(C-\gamma_{L I}\right)}{\gamma_{S R} \gamma_{L I} \gamma_{R D}+C\left(C+\gamma_{R D}-\gamma_{L I}\right)}, & \gamma_{L I}<C \\
0, & \gamma_{L I} \geqslant C
\end{array}\right.
$$

with $C=\bar{\gamma}_{S R}+\bar{\gamma}_{L I}+1$. Here, $\gamma_{L I}<C$ is obtained from the condition $\beta^{2}<1 /\left(P_{R}\left|h_{L I}\right|^{2}\right)$ given before. Note that when the condition $\beta^{2}<1 /\left(P_{R}\left|h_{L I}\right|^{2}\right)$ is not satisfied, i.e., when $\gamma_{L I} \geqslant C$, then $\gamma_{\text {end }}=0$ since the power of the interference goes to infinity.

In order to make a fair comparison, average power consumption of the fixed-gain AF-FD relays should be taken into account. By using (3) and $\beta$, the average transmission power at the relay is given by

$$
E\left[\left\langle P_{R}|s(t)|^{2}\right\rangle\right]=P_{R} E\left[\frac{\gamma_{S R}+1}{C-\gamma_{L I}}\right] .
$$

By using the independence property of $\gamma_{S R}$ and $\gamma_{L I}$, the average power consumption can be obtained as

$E\left[\left\langle P_{R}|s(t)|^{2}\right\rangle\right]=P_{R} \frac{E\left[\gamma_{S R}+1\right]}{E\left[C-\gamma_{L I}\right]}=P_{R} \frac{\bar{\gamma}_{S R}+1}{C-\bar{\gamma}_{L I}}=P_{R}$.

Although the instantaneous transmission power at the relay varies for the fixed-gain AF-FD relaying, the average value is set to $P_{R}$ due to the selected $\beta$. It is important to note that we assume the dynamic range of the power amplifier at the relay is unrestricted for the fixed-gain AF-FD systems similar to [8] and [21].

\subsection{Variable-gain relaying}

In order to assure the instantaneous transmission power as $P_{R}$, the amplification factor is set to $\beta=1 / \sqrt{P_{S}\left|h_{S R}\right|^{2}+P_{R}\left|h_{L I}\right|^{2}+\sigma_{R}^{2}}$. Please note that the same amplification factor is commonly used in the FD literature such as in $[12,14,18-20]$. Although the amplification factor changes with the instantaneous value of residual LI channel coefficient, which is an unknown for the relay, $\left|h_{L I}\right|^{2}$ can be obtained by using the energy detection techniques as mentioned in [14]. Then, the instantaneous SINR becomes

$$
\gamma_{\text {end }}=\frac{X_{1} \gamma_{R D}}{X_{1}+\gamma_{R D}+1}
$$

where $X_{1}=\gamma_{S R} /\left(\gamma_{L I}+1\right)$. Note that the condition $\beta^{2}<1 /\left(P_{R}\left|h_{L I}\right|^{2}\right)$ given before is always satisfied for the variable-gain relaying. By substituting $\beta$ into (3), the instantaneous transmission power for the variable-gain AF-FD relaying can be easily given by [3]

$$
\left\langle P_{R}|s(t)|^{2}\right\rangle=P_{R}
$$

which also shows that the average transmission power of the relay for both the fixed-gain and variable-gain AF-FD relaying are the same and equal to $P_{R}$.

\section{Exact outage probability analysis}

In this section, exact outage probabilities of the considered FD systems are analyzed by using the CDFs of $\gamma_{\text {end }}$ 
given in (5) and (8). The exact outage probability equals $P_{\text {out }}=F_{\gamma_{\text {end }}}\left(\gamma_{\text {th }}\right)$ where $F_{\gamma_{\text {end }}}($.$) is CDF of \gamma_{\text {end }}$ and $\gamma_{t h}$ is a threshold SINR.

\subsection{Fixed-gain relaying}

The CDF expression for the SINR given in (5) can be expressed as

$$
\begin{aligned}
F_{\gamma_{\text {end }}}(x)= & P\left(\gamma_{L I}<C\right) F_{\gamma_{\text {end }}}\left(x \mid \gamma_{L I}<C\right) \\
& +P\left(\gamma_{L I} \geqslant C\right) F_{\gamma_{\text {end }}}\left(x \mid \gamma_{L I} \geqslant C\right)
\end{aligned}
$$

where $P\left(\gamma_{L I}<C\right)=F_{\gamma_{L I}}(C)=\frac{\gamma\left(m_{L I}, \mu_{L I} C\right)}{\Gamma\left(m_{L I}\right)}$ and therefore $P\left(\gamma_{L I} \geqslant C\right)=1-F_{\gamma_{L I}}(C)$ for non-identical Nakagami- $m$ fading channels. In (10), the conditional CDF $F_{\gamma_{\text {end }}}\left(x \mid \gamma_{L I} \geqslant C\right)=P\left(\gamma_{\text {end }}<x \mid \gamma_{L I} \geqslant C\right)=1$ since $\gamma_{\text {end }}=0$ when $\gamma_{L I} \geqslant C$. Hence, we obtain

$$
P_{\text {out }}=1-\frac{\gamma\left(m_{L I}, \mu_{L I} C\right)}{\Gamma\left(m_{L I}\right)}\left[1-F_{\gamma_{\text {end }}}\left(\gamma_{t h} \mid \gamma_{L I}<C\right)\right]
$$

where the conditional CDF $F_{\gamma_{\text {end }}}\left(\gamma_{t h} \mid \gamma_{L I}<C\right)$ can be given by

$$
\begin{aligned}
F_{\gamma_{\text {end }}}\left(x \mid \gamma_{L I}<C\right) & =P\left(\frac{\gamma_{S R} \gamma_{R D}\left(C-\gamma_{L I}\right)}{\gamma_{S R} \gamma_{L L} \gamma_{R D}+C\left(C+\gamma_{R D}-\gamma_{L I}\right)}<x \mid \gamma_{L I}<C\right) \\
& =\int_{z=0}^{C} \int_{y=0}^{\infty} P\left(\frac{\gamma_{S R} y(C-z)}{\gamma_{S R} y z+C(C+y-z)}<x\right) p_{\gamma_{R D}}(y) p_{\gamma_{L I}}(z) d y d z .
\end{aligned}
$$

In the above integral, the probability term can be written as

$$
\begin{aligned}
P\left(\frac{\gamma_{S R} y(C-z)}{\gamma_{S R} y z+C(C+y-z)}<x\right) & =P\left(\gamma_{S R} y(C-z(x+1))<x C(C+y-z)\right) \\
& = \begin{cases}F_{\gamma_{S R}}\left(\frac{x C(C+y-z)}{y(C-z(x+1))}\right), & \text { if } z<\frac{C}{x+1} \\
1, & \text { if } z \geqslant \frac{C}{x+1} .\end{cases}
\end{aligned}
$$

By substituting (13) into (12), $F_{\gamma_{\text {end }}}\left(x \mid \gamma_{L I}<C\right)$ is rewritten as

$$
\begin{aligned}
F_{\gamma_{\text {end }}}\left(x \mid \gamma_{L I}<C\right)= & \left.\int_{z=0}^{\frac{C}{x+1}} \int_{y=0}^{\infty}\left[1-\bar{F}_{\gamma_{S R}}\left(\frac{x C(C+y-z)}{y(C-z(x+1))}\right)\right]\right]_{\gamma_{R D}(y) p_{\gamma_{L I}}(z) d y d z} \\
& +\int_{z=\frac{C}{x+1}}^{C} \int_{y=0}^{\infty} p_{\gamma_{R D}}(y) p_{\gamma_{L I}}(z) d y d z
\end{aligned}
$$

where $\bar{F}_{\gamma_{S R}}()=.1-F_{\gamma_{S R}}($.$) . So, F_{\gamma_{\text {end }}}\left(x \mid \gamma_{L I}<C\right)$ is written as

$$
\begin{aligned}
& F_{\gamma_{\text {end }}}\left(x \mid \gamma_{L I}<C\right)=\overbrace{\int_{z=0}^{C} \int_{y=0}^{\infty} p_{\gamma_{R D}}(y) p_{\gamma_{L I}}(z) d y d z}^{I_{1}} \\
&-\underbrace{\int_{z=0}^{\frac{C}{x+1}} \int_{y=0}^{\infty} \bar{F}_{\gamma_{S R}}\left(\frac{x C(C+y-z)}{y(C-z(x+1))}\right) p_{\gamma_{R D}}(y) p_{\gamma_{L I}}(z) d y d z}_{I_{2}} .
\end{aligned}
$$

In the above equation, the first integral term $I_{1}$ can be simply solved as

$I_{1}=\int_{z=0}^{C} \int_{y=0}^{\infty} p_{\gamma_{R D}}(y) p_{\gamma_{L I}}(z) d y d z=F_{\gamma_{L I}}(C)=\frac{\gamma\left(m_{L I}, \mu_{L I} C\right)}{\Gamma\left(m_{L I}\right)}$.

The second integral term $I_{2}$ can be calculated as

$$
\begin{aligned}
I_{2}= & \frac{\mu_{R D}^{m_{R D}} \mu_{L I}^{m_{L I}}}{\Gamma\left(m_{S R}\right) \Gamma\left(m_{R D}\right) \Gamma\left(m_{L I}\right)} \int_{z=0}^{\frac{C}{x+1}} z^{m_{L I}-1} e^{-\mu_{L I}} \\
& \times \int_{y=0}^{\infty}\left[\Gamma\left(m_{S R}\right)-\gamma\left(m_{S R}, \frac{x \mu_{S R} C(C+y-z)}{y(C-z(x+1))}\right)\right] y^{m_{R D}-1} e^{-\mu_{R D} y} d y d z .
\end{aligned}
$$

For integer values of $m_{S R}$, by using ([22] eq. (8.352.6)), $I_{2}$ can be rewritten as

$$
\begin{aligned}
I_{2}= & \frac{\mu_{R D}^{m_{R D}} \mu_{L I}^{m_{L I}}}{\Gamma\left(m_{R D}\right) \Gamma\left(m_{L I}\right)} \sum_{k=0}^{m_{S R}-1} \frac{\left(x \mu_{S R} C\right)^{k}}{k !} \int_{z=0}^{\frac{C}{x+1}} z^{m_{L I}-1} e^{-\mu_{L I} z-\frac{x \mu_{S R} C}{C-z(x+1)}} \\
& \times \int_{y=0}^{\infty} e^{-\frac{x \mu_{S R} C(C-z)}{y(C-z(x+1))}}\left[\frac{C+y-z}{y(C-z(x+1))}\right]^{k} y^{m_{R D}-1} e^{-\mu_{R D} y} d y d z .
\end{aligned}
$$

By using the binomial equation for the term shown in the square brackets the above equation, we obtain

$$
\begin{aligned}
I_{2}= & \frac{\mu_{R D}^{m_{R D}} \mu_{L I}^{m_{L I}}}{\Gamma\left(m_{R D}\right) \Gamma\left(m_{L I}\right)} \sum_{k=0}^{m_{S R}-1} \sum_{i=0}^{k} \frac{\left(x \mu_{S R} C\right)^{k}}{i !(k-i) !} \int_{z=0}^{\frac{C}{x+1}} \frac{(C-z)^{i}}{(C-z(x+1))^{k}} \\
& \times z^{m_{L I}-1} e^{-\mu_{L I} z-\frac{x \mu_{S R} C}{C-z(x+1)}} \underbrace{\int_{y=0}^{\infty} e^{-\frac{x \mu_{S R} C(C-z)}{y(C-z(x+1))}} y^{m_{R D}-1-i} e^{-\mu_{R D} y} d y d z .}_{I_{3}}
\end{aligned}
$$

By using ([22] eq. (3.471.9)), the integral term $I_{3}$ is solved as

$$
I_{3}=2\left(\frac{x C(C-z) \mu_{S R}}{(C-z(x+1)) \mu_{R D}}\right)^{\frac{m_{R D}-i}{2}} K_{m_{R D}-i}\left(2 \sqrt{\frac{x C(C-z) \mu_{S R} \mu_{R D}}{C-z(x+1)}}\right)
$$


where $K_{m_{R D}-i}($.$) is the \left(m_{R D}-i\right)^{t h}$ order modified Bessel function of second kind ([22] eq. (8.407.1)). Using (20) in (19) gives

$$
\begin{aligned}
I_{2}= & \frac{2}{\Gamma\left(m_{R D}\right) \Gamma\left(m_{L I}\right)}\left(\gamma_{t h} \mu_{S R} \mu_{R D} C\right)^{\frac{m_{R D}}{2}} \mu_{L I}^{m_{L I}} \\
& \times \sum_{k=0}^{m_{S R}-1} \sum_{i=0}^{k}\left(\gamma_{t h} \mu_{S R} C\right)^{\frac{2 k-i}{2}} \frac{1}{i !(k-i) !} \mu_{R D}^{i / 2} \\
& \times \int_{z=0}^{\frac{C}{\gamma_{t h}+1}} \frac{(C-z)^{\frac{m_{R D}+i}{2}} z^{m_{L I}-1}}{\left(C-z\left(\gamma_{t h}+1\right)\right)^{\frac{m_{R D}-i+2 k}{2}}} e^{-\mu_{L I} z-\frac{\gamma_{t h} \mu_{S R} C}{C-z\left(\gamma_{t h}+1\right)}} \\
& \times K_{m_{R D}-i}\left(2 \sqrt{\frac{\gamma_{t h} \mu_{S R} \mu_{R D} C(C-z)}{C-z\left(\gamma_{t h}+1\right)}}\right) d z .
\end{aligned}
$$

To the best of authors' knowledge, there is no closedform solution for the above expression. By substituting (15), (16) and (21) into (11), $P_{\text {out }}$ for the fixed-gain AF-FD relaying can be evaluated by using numerical integration and it is rewritten as

$$
P_{o u t}=1-\frac{\gamma\left(m_{L I}, \mu_{L I} C\right)}{\Gamma\left(m_{L I}\right)}\left[1-\frac{\gamma\left(m_{L I}, \mu_{L I} C\right)}{\Gamma\left(m_{L I}\right)}+I_{2}\right] .
$$

The above expression is in a single-integral form. Therefore, it can be easily and efficiently evaluated with common mathematical software packages such as MATLAB, MATHEMATICA or MAPLE.

\subsection{Variable-gain relaying}

The CDF expression for the SINR given in (8) is given by

$$
\begin{aligned}
F_{\gamma_{\text {end }}}(x) & =P\left(\gamma_{\text {end }}<x\right)=P\left(\frac{X_{1} \gamma_{R D}}{X_{1}+\gamma_{R D}+1}<x\right) \\
& =\int_{y=0}^{\infty} P\left(\frac{X_{1} y}{X_{1}+y+1}<x\right) p_{\gamma_{R D}}(y) d y .
\end{aligned}
$$

By using ([18] eq. (13)), $F_{\gamma_{\text {end }}}(x)$ can be rewritten as

$$
\begin{aligned}
F_{\gamma_{\text {end }}}(x) & =F_{\gamma_{R D}}(x)+\int_{y=x}^{\infty} F_{X_{1}}\left(\frac{x(y+1)}{y-x}\right) p_{\gamma_{R D}}(y) d y \\
& =1-\int_{u=0}^{\infty} \bar{F}_{X_{1}}\left(\frac{x(u+x+1)}{u}\right) p_{\gamma_{R D}}(u+x) d u
\end{aligned}
$$

where $\bar{F}_{X_{1}}()=.1-F_{X_{1}}($.$) . Again by using ([18] eq. (14)),$

$$
\begin{aligned}
F_{X_{1}}(z) & =\int_{v=0}^{\infty} F_{\gamma_{S R}}(z(v+1)) p_{\gamma_{L I}}(v) d v \\
& =\frac{\mu_{L I}^{m_{L I}}}{\Gamma\left(m_{S R}\right) \Gamma\left(m_{L I}\right)} \int_{v=0}^{\infty} \gamma\left(m_{S R}, \mu_{S R} z(v+1)\right) v^{m_{L I}-1} e^{-\mu_{L I} v} d v
\end{aligned}
$$

can be obtained. For integer values of $m_{S R}$, by using ([22], eq. (8.352.6)), ([23] eq. (8)) and also the binomial expansion,

$\bar{F}_{X_{1}}(z)=\frac{\mu_{L I}^{m_{L I}} e^{-\mu_{S R} z}}{\Gamma\left(m_{L I}\right)} \sum_{k=0}^{m_{S R}-1} \sum_{b=0}^{k} \frac{\Gamma\left(b+m_{L I}\right)\left(\mu_{S R} z\right)^{k}}{b !(k-b) !\left(\mu_{L I}+\mu_{S R} z\right)^{b+m_{L I}}}$

can be obtained. Now substituting (26) into (24), $P_{\text {out }}$ for the variable-gain AF-FD relaying is

$$
\begin{aligned}
P_{\text {out }} & =1-\frac{e^{-\left(\mu_{S R}+\mu_{R D}\right) \gamma_{t h}} \mu_{R D}^{m_{R D}} \mu_{L I}^{m_{L I}}}{\Gamma\left(m_{R D}\right) \Gamma\left(m_{L I}\right)} \sum_{k=0}^{m_{S R}-1} \sum_{b=0}^{k} \frac{\Gamma\left(b+m_{L I}\right)}{b !(k-b) !} \\
& \times\left(\mu_{S R} \gamma_{t h}\right)^{k} \int_{u=0}^{\infty} e^{-\mu_{R D} u-\mu_{S R} \frac{\left(\gamma_{t h}^{2}+\gamma_{t h}\right)}{u}}\left[\frac{u+\gamma_{t h}+1}{u}\right]^{k} \\
& \times\left(u+\gamma_{t h}\right)^{m_{R D}-1}\left[\mu_{L I}+\mu_{S R} \frac{\gamma_{t h}\left(u+\gamma_{t h}+1\right)}{u}\right]^{-b-m_{L I}} d u .
\end{aligned}
$$

The above expression is in single-integral form as well. Hence, it can be also easily and efficiently evaluated with common mathematical software packages such as MATLAB, MATHEMATICA, or MAPLE. As mentioned in the Introduction, outage performance of the variable-gain AF-FD relaying systems in Nakagami- $m$ fading channels is also independently evaluated in [20]. On the other hand, the exact outage probability expression given in ([20] eq. (16)) is written in terms of EGBMGF which contains MellinBarnes type double-contour-integration ([24] Table I). Although, MATHEMATICA implementation of the EGBMGF function has been provided in ([24] Table II), the built-in function is not available for EGBMGF to the best of our knowledge. Also, different from [20], our result is valid for both integer and non-integer $m_{R D}$ values.

\section{Lower-bound and asymptotic outage probability analyses}

In this section, both the lower-bound and asymptotic outage probability of the fixed-gain and variable-gain AF-FD systems are investigated. 


\subsection{Fixed-gain relaying}

\subsubsection{Lower-bound}

In the previous section, the exact outage probability of the fixed-gain AF-FD relaying is derived in a single-integral form and given in (22). One can show that the integral term at (21) can be upper-bounded when $C-z\left(\gamma_{t h}+1\right)$ term inside the integration is replaced with $C-z$ term. This leads to a lower-bound of the outage probability which can be given by

$$
\begin{aligned}
& P_{\text {out }, \text { low }}=1-\frac{\gamma\left(m_{L I}, \mu_{L I} C\right)}{\Gamma\left(m_{L I}\right)}\left[1-\frac{\gamma\left(m_{L I}, \mu_{L I} C\right)}{\Gamma\left(m_{L I}\right)}\right. \\
& +\frac{2\left(\gamma_{t h} \mu_{S R} \mu_{R D} C\right)^{\frac{m_{R D}}{2}} \mu_{L I}^{m_{L I}}}{\Gamma\left(m_{R D}\right) \Gamma\left(m_{L I}\right)} \sum_{k=0}^{m_{S R}-1} \sum_{i=0}^{k} \frac{\mu_{R D}^{i / 2}}{i !(k-i) !} \\
& \times\left(\gamma_{t h} \mu_{S R} C\right)^{\frac{2 k-i}{2}} K_{m_{R D}-i}\left(2 \sqrt{\gamma_{t h} \mu_{S R} \mu_{R D} C}\right) \\
& \times \underbrace{\left.\int_{z=0}^{\frac{C}{\gamma_{t h}+1}}(C-z)^{i-k} z^{m_{L I}-1} e^{-\mu_{L I} z-\frac{\gamma_{t h} \mu_{S R} C}{C-z}} d z\right]}_{I_{4}} .
\end{aligned}
$$

By applying the variable transformation as $t=\frac{C-z}{C}$ and using the binomial expansion, the above integral $I_{4}$ is expressed as

$I_{4}=\sum_{b=0}^{m_{L I}-1}\left(\begin{array}{c}m_{L I}-1 \\ b\end{array}\right) \frac{C^{m_{L I}+i-k}}{e^{\mu_{L I} C}(-1)^{-b}} \int_{t=\frac{\gamma_{t h}}{\gamma_{t h}+1}}^{1} \frac{t^{i+b-k}}{e^{-\mu_{L I} C t+\frac{\gamma_{t h} \mu_{S R}}{t}}} d t$.

Then, the power series and the binomial expansions are used respectively as follows

$$
\begin{aligned}
I_{4} & =\frac{C^{m_{L I}+i-k}}{e^{\mu_{L I} C}} \sum_{b=0}^{m_{L I}-1} \sum_{n=0}^{\infty}\left(\begin{array}{c}
m_{L I}-1 \\
b
\end{array}\right) \frac{(-1)^{b}}{n !} \\
& \times \int_{t=\frac{\gamma_{t h}}{\gamma_{t h}+1}} t^{i+b-k}\left(\frac{\gamma_{t h} \mu_{S R}}{t}-\mu_{L I} C t\right)^{n} d t \\
& =\frac{C^{m_{L I}+i-k}}{e^{\mu_{L I} C}} \sum_{b=0}^{m_{L I}-1} \sum_{n=0}^{\infty} \sum_{u=0}^{n}\left(\begin{array}{c}
m_{L I}-1 \\
b
\end{array}\right) \frac{(-1)^{b+u}}{(n-u) ! u !} \\
& \times \frac{\left(\gamma_{t h} \mu_{S R}\right)^{u}}{\left(\mu_{L I} C\right)^{u-n}} \underbrace{1}_{\int_{t=\frac{\gamma_{t h}}{\gamma_{t h}+1}}^{1} t^{i+b+n-k-2 u} d t}
\end{aligned}
$$

where $I_{5}$ can be easily solved as

$$
I_{5}= \begin{cases}\ln \left(\frac{\gamma_{t h}+1}{\gamma_{t h}}\right), & \text { if } n+b+i-k-2 u=-1 \\ \frac{1}{\gamma_{t h}+1}, & \text { if } n+b+i-k-2 u=0 \\ \frac{1-\left[\gamma_{t h} /\left(\gamma_{t h}+1\right)\right]^{n+b+i-k-2 u+1}}{n+b+i-k-2 u+1}, & \text { otherwise. }\end{cases}
$$

By combining (28), (30), and (31), we obtain the lowerbound expression for the fixed-gain AF-FD systems in closed-form as

$$
\begin{aligned}
P_{\text {out }, \text { low }}= & 1-\frac{\gamma\left(m_{L I}, \mu_{L I} C\right)}{\Gamma\left(m_{L I}\right)}\left[1-\frac{\gamma\left(m_{L I}, \mu_{L I} C\right)}{\Gamma\left(m_{L I}\right)}\right. \\
& +\frac{2 e^{-\mu_{L I} C}}{\Gamma\left(m_{R D}\right) \Gamma\left(m_{L I}\right)} \sum_{k=0}^{m_{S R}-1} \sum_{i=0}^{k} \sum_{b=0}^{m_{L I}-1} \sum_{n=0}^{\infty} \sum_{u=0}^{n}\left(\begin{array}{c}
m_{L I}-1 \\
b
\end{array}\right) \\
& \times \frac{\left(\gamma_{t h} \mu_{S R}\right)^{\frac{m_{R D}-i}{2}}+k+u}{i !(k-i) !(n-u) ! u !(-1)^{-b-u}} \mu_{L I}^{\frac{m_{R D}+i}{2}} C^{m_{L I}+\frac{m_{R D}+i}{2}+n-u} \\
& \left.\left.\times K_{m_{R D}-i}\left(2 \sqrt{\gamma_{t h} \mu_{S R} \mu_{R D} C}\right) I_{5}\right)\right]
\end{aligned}
$$

\subsubsection{Asymptotic outage probability}

When signal-to-noise ratio (SNR) goes to infinity, i.e., when $P_{S}, P_{R} \rightarrow \infty$ for the finite $\sigma_{R}^{2}$ and $\sigma_{D}^{2}$, the instantaneous SINR in (5) becomes

$$
\begin{aligned}
\lim _{\substack{P_{S} \rightarrow \infty \\
P_{R} \rightarrow \infty}} \gamma_{\text {end }} & =\lim _{\substack{P_{S} \rightarrow \infty \\
P_{R} \rightarrow \infty}} \frac{\gamma_{S R} \gamma_{R D}\left(C-\gamma_{L I}\right)}{\gamma_{S R} \gamma_{L I} \gamma_{R D}+C\left(C+\gamma_{R D}-\gamma_{L I}\right)} \\
& =\lim _{\substack{P_{S} \rightarrow \infty \\
P_{R} \rightarrow \infty}} \frac{C-\gamma_{L I}}{\gamma_{L I}}=\frac{\bar{\gamma}_{S R}+\bar{\gamma}_{L I}}{\gamma_{L I}}-1 .
\end{aligned}
$$

The asymptotic expression for the outage probability of the fixed-gain AF-FD systems can be obtained from (11). One can easily show that $\gamma\left(m_{L I}, \mu_{L I} C\right)$ goes to $\gamma\left(m_{L I}, m_{L I}(\eta+1)\right)$ for $P_{S}, P_{R} \rightarrow \infty$ where $\eta=\bar{\gamma}_{S R} / \bar{\gamma}_{L I}$. The conditional CDF term in (11) is obtained for the asymptotic SINR as

$$
\begin{aligned}
F_{\gamma_{e n d}}^{\infty}\left(x \mid \gamma_{L I}<C\right) & =P\left(\frac{\bar{\gamma}_{S R}+\bar{\gamma}_{L I}}{\gamma_{L I}}-1<x \mid \gamma_{L I}<C\right) \\
& =\frac{P\left(\frac{\bar{\gamma}_{S R}+\bar{\gamma}_{L I}}{x+1}<\gamma_{L I}<C\right)}{P\left(\gamma_{L I}<C\right)} \\
& =1-\frac{F_{\gamma_{L I}}\left(\frac{\bar{\gamma}_{S R}+\bar{\gamma}_{L I}}{x+1}\right)}{F_{\gamma_{L I}}(C)} . \\
& =1-\frac{\gamma\left(m_{L I}, m_{L I}(\eta+1) /\left(\gamma_{t h}+1\right)\right)}{\gamma\left(m_{L I}, m_{L I}(\eta+1)\right)}
\end{aligned}
$$




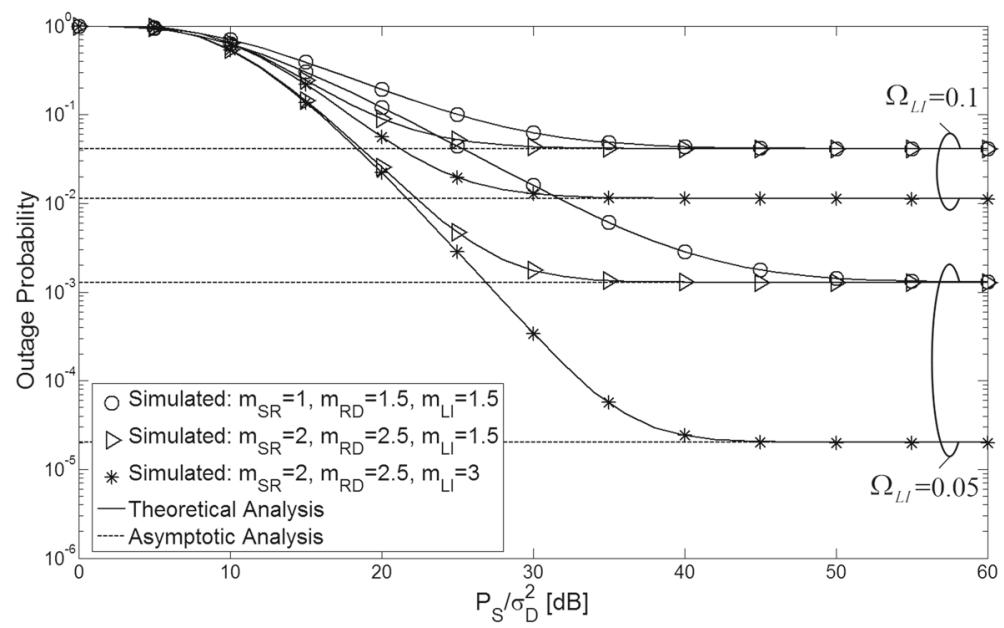

Fig. 2 Outage probability of the fixed-gain AF-FD systems for $\Omega_{L l}=\{0.05,0.1\}, \gamma_{t h}=3$ and various $m$ values

By substituting (34) in (11), the asymptotic outage probability is obtained in closed-form as

$$
P_{\text {out }}^{\infty}=1-\frac{\gamma\left(m_{L I}, m_{L I}(\eta+1) /\left(\gamma_{t h}+1\right)\right)}{\Gamma\left(m_{L I}\right)} .
$$

As seen, $P_{\text {out }}^{\infty}$ for the fixed-gain case is a constant and therefore leads to zero diversity order at high SNR and does not depend on $m_{S R}, m_{R D}$ and $\Omega_{R D}$ parameters.

\subsection{Variable-gain relaying}

\subsubsection{Lower-bound}

In order to obtain a lower-bound for the variablegain AF-FD relaying systems, we use the property of $\gamma_{\text {end }}<X_{1} \gamma_{R D} /\left(X_{1}+\gamma_{R D}\right)<\min \left(X_{1}, \gamma_{R D}\right)$ where the instantaneous SINR for variable-gain relaying is given in (8). So, the lower-bound can be expressed as

$$
\begin{aligned}
P_{\text {out }, \text { low }} & =P\left(\min \left\{X_{1}, \gamma_{R D}\right\} \leq \gamma_{t h}\right) \\
& =1-P\left(\min \left\{X_{1}, \gamma_{R D}\right\}>\gamma_{t h}\right) \\
& =1-\bar{F}_{X_{1}}\left(\gamma_{t h}\right) \bar{F}_{\gamma_{R D}}\left(\gamma_{t h}\right)
\end{aligned}
$$

where $\bar{F}_{X_{1}}$ (.) was already found in (26). Therefore, we can easily derive the lower-bound expression in closedform as

$$
\begin{aligned}
P_{\text {out }, \text { low }}= & 1-\frac{\Gamma\left(m_{R D}, \gamma_{t h} \mu_{R D}\right) \mu_{L I}^{m_{L I}}}{\Gamma\left(m_{R D}\right) \Gamma\left(m_{L I}\right) e^{\mu_{S R} \gamma_{t h}}} \\
& \times \sum_{k=0}^{m_{S R}-1} \sum_{b=0}^{k} \frac{\Gamma\left(b+m_{L I}\right)\left(\mu_{S R} \gamma_{t h}\right)^{k}}{b !(k-b) !\left(\mu_{L I}+\mu_{S R} \gamma_{t h}\right)^{b+m_{L I}}}
\end{aligned}
$$

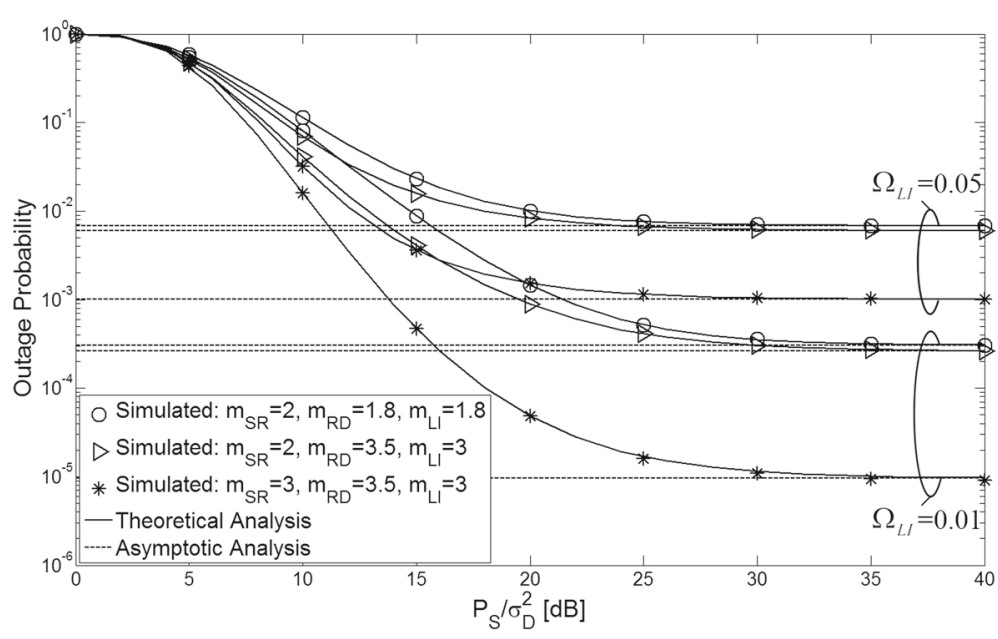

Fig. 3 Outage probability of the variable-gain AF-FD systems for $\Omega_{L l}=\{0.01,0.05\}, \gamma_{\text {th }}=1$ and various $m$ values 


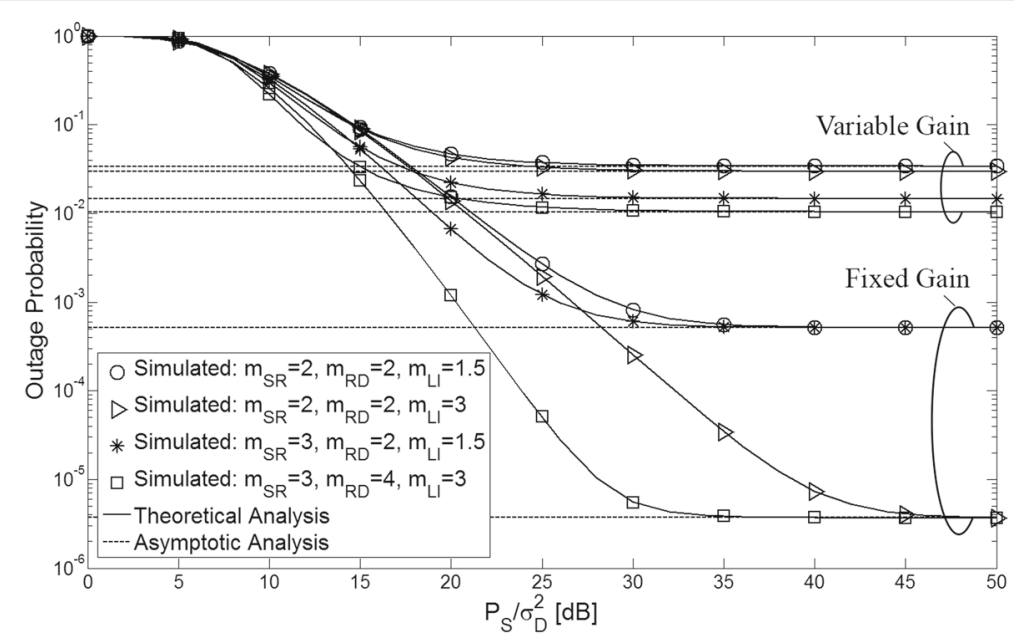

Fig. 4 Outage probability of the fixed-gain and variable-gain AF-FD systems for $\Omega_{L l}=0.06, \gamma_{t h}=2$ and various $m$ values

\subsubsection{Asymptotic outage probability}

When $P_{S}, P_{R} \rightarrow \infty$, the instantaneous SINR in (8) becomes

$$
\lim _{\substack{P_{S} \rightarrow \infty \\ P_{R} \rightarrow \infty}} \gamma_{\text {end }}=\lim _{\substack{P_{S} \rightarrow \infty \\ P_{R} \rightarrow \infty}} \frac{X_{1} \gamma_{R D}}{X_{1}+\gamma_{R D}+1}=\frac{\gamma_{S R}}{\gamma_{L I}}
$$

In that case, the corresponding asymptotic CDF can be given by $F_{\gamma_{\text {end }}}^{\infty}(x)=P\left(\gamma_{S R} / \gamma_{L I}<x\right)=$ $\int_{0}^{\infty} F_{\gamma_{S R}}(x y) p_{\gamma_{L I}}(y) d y$. For integer values of $m_{S R}$ by using ([22] eq. (8.352.6)), the asymptotic CDF expression is rewritten as

$$
\begin{aligned}
F_{\gamma_{\text {end }}}^{\infty} & =\int_{y=0}^{\infty}\left[1-e^{-x y \mu_{S R}} \sum_{i=0}^{m_{S R}-1} \frac{\left(x y \mu_{S R}\right)^{i}}{i !}\right] \frac{\mu_{L I}^{m_{L I}} y^{m_{L I}-1} e^{-y \mu_{L I}}}{\Gamma\left(m_{L I}\right)} d y \\
& =1-\frac{\mu_{L I}^{m_{L I}}}{\Gamma\left(m_{L I}\right)} \sum_{i=0}^{m_{S R}-1} \frac{\left(x \mu_{S R}\right)^{i}}{i !} \int_{y=0}^{\infty} y^{m_{L I}+i-1} e^{-y\left(\mu_{L I}+x \mu_{S R}\right)} d y .
\end{aligned}
$$

The above integral can be easily solved by using ([22] eq. (3.351.3)). At the end, we obtain the asymptotic outage probability in closed-form as

$$
\begin{aligned}
P_{\text {out }}^{\infty}= & 1-\frac{1}{\Gamma\left(m_{L I}\right)}\left(1+\frac{\gamma_{t h} \mu_{S R}}{\mu_{L I}}\right)^{-m_{L I}} \\
& \times \sum_{i=0}^{m_{S R}-1} \frac{\Gamma\left(m_{L I}+i\right)}{i !}\left(1+\frac{\mu_{L I}}{\gamma_{t h} \mu_{S R}}\right)^{-i} .
\end{aligned}
$$

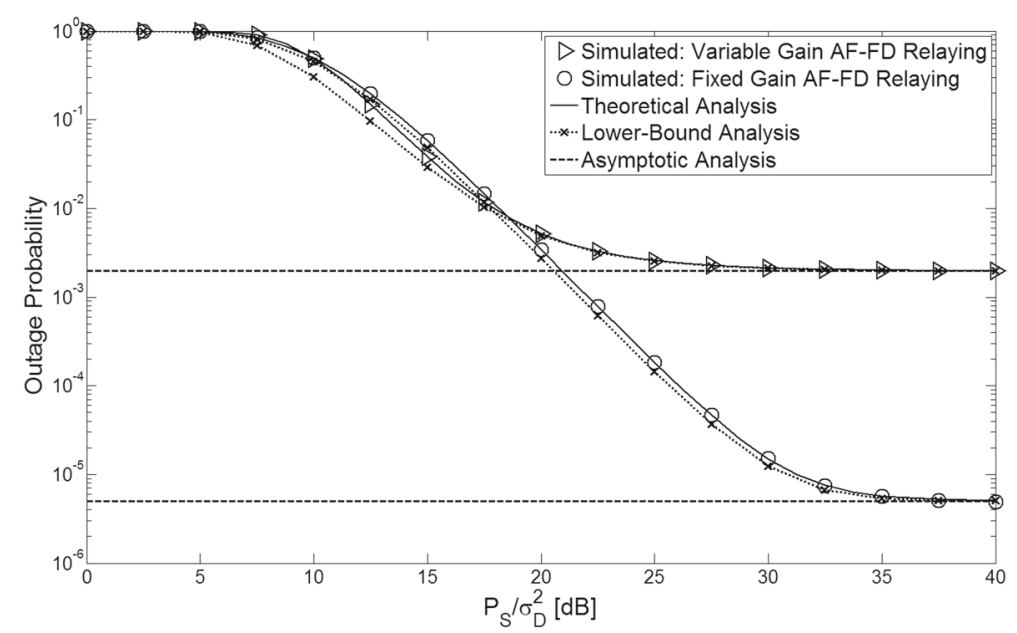

Fig. 5 Outage probability of the fixed-gain and variable-gain AF-FD systems for $2 P_{S}=3 P_{R}, \Omega_{S R}=1, \Omega_{R D}=2, \Omega_{L I}=0.025, m_{S R}=4, m_{R D}=2.5$, $m_{L l}=1$ and $\gamma_{\text {th }}=4$ 


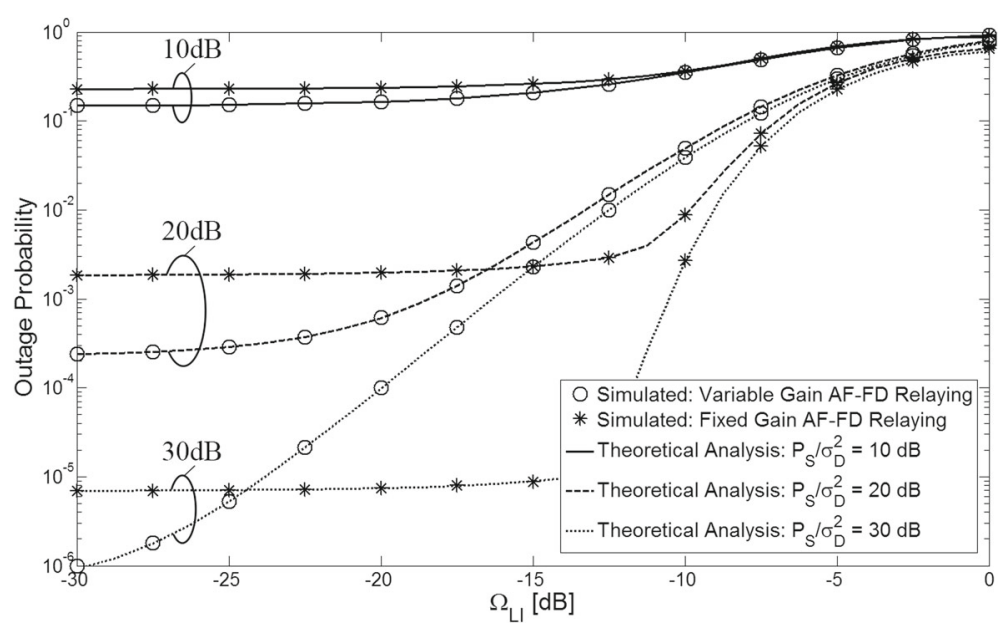

Fig. 6 Outage probability of the fixed-gain and variable-gain AF-FD systems for $m_{S R}=3, m_{R D}=m_{L I}=2.5$ and $\gamma_{\text {th }}=2$

As seen, $P_{o u t}^{\infty}$ for the variable-gain case is also a constant, hence leads to zero diversity order at high SNR. In addition, it does not depend on $m_{R D}$ and $\Omega_{R D}$ parameters. Note that similar expression was also obtained independently in ([20] eq. (22)). Here, we provide (40) for the sake of consistency.

\section{Numerical results}

The theoretical (numerical) and Monte-Carlo simulations outage performance results of the fixed-gain and variablegain AF-FD systems are shown in this section. During the outage performance analyses, we assume that $\sigma_{D}^{2}=\sigma_{R}^{2}$ for all the scenarios and $\Omega_{S R}=\Omega_{R D}=1, P_{S}=P_{R}$ unless otherwise stated. Moreover, the residual LI power, apart from Fig. 6, is chosen in the range of 0.01 and 0.1 ( -20 and $-10 \mathrm{~dB})$ as in $[12,14,18-20]$.

Figure 2 plots the outage probability results of the fixedgain AF-FD relaying versus $P_{S} / \sigma_{D}^{2}$ for two different LI powers, $\Omega_{L I}=\{0.05,0.1\}$, and various $m$ values. The threshold SINR was set to $\gamma_{t h}=3$ for these scenarios. As seen from the curves, the outage probability decreases as $\Omega_{L I}$ decreases, i.e., efficiency of the loop-interference cancellation process increases. As seen, the exact analytical results obtained from (22) perfectly overlap with the simulation results for all $P_{S} / \sigma_{D}^{2}$ values, verifying the accuracy of our analyses. Moreover, the error floors monitored in simulations are consistent with the asymptotic expression for the fixed-gain AF-FD relaying given in (35). We see

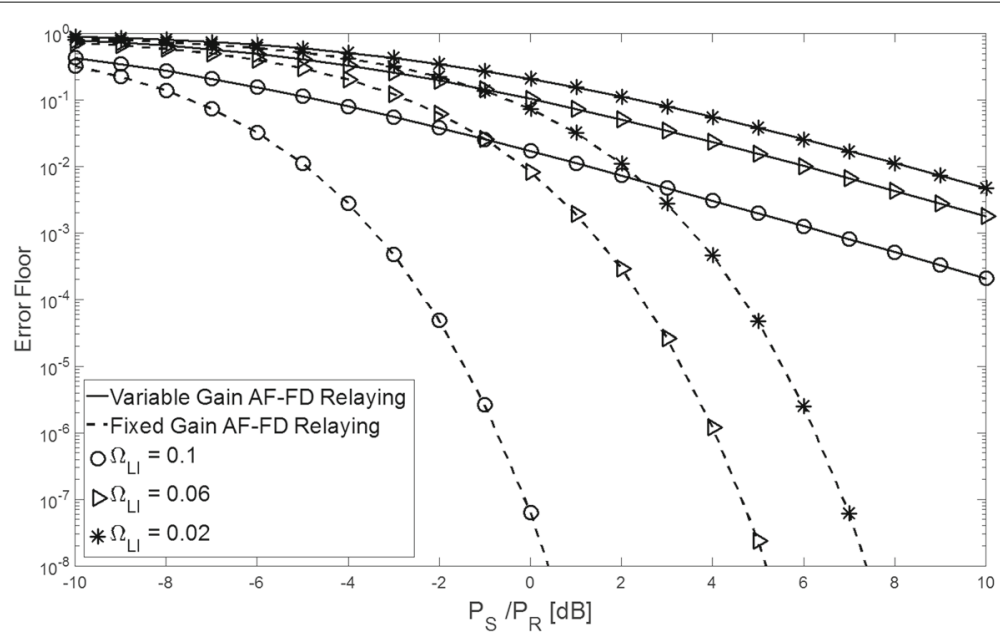

Fig. 7 Error floor of the fixed-gain and variable-gain AF-FD systems for $\Omega_{S R}=1.2, \Omega_{R D}=0.8, \Omega_{L I}=\{0.02,0.06,0.1\}, m_{S R}=2, m_{R D}=1.4, m_{L I}=1.9$ and $\gamma_{\text {th }}=5$ 
that the dominant $m$ parameter on the asymptotic performance is $m_{L I}$ and other $m$ parameters have no effect on the error floor for the fixed-gain AF-FD relaying.

The outage probability results of the variable-gain AFFD relaying for $\Omega_{L I}=\{0.01,0.05\}, \gamma_{t h}=1$ and various $m$ values are demonstrated by Fig. 3 . The simulation results are perfectly matched with both the analytical exact and the asymptotic outage results given by (27) and (40), respectively. Different from the fixed-gain AF-FD relaying, we see that the parameter $m_{S R}$ has more dominant effect than the other $m$ parameters on the asymptotic performance in the case of variable-gain AF-FD relaying.

Figure 4 compares the outage performance for the fixedgain and variable-gain AF-FD systems for $\Omega_{L I}=0.06$, $\gamma_{t h}=2$ and various $m$ values. As seen, the fixed-gain AF-FD relaying provides better outage performance than the variable-gain AF-FD relaying at moderate and high SINR regions since the LI effect decreases due to the fixed amplification factor. We also present the asymptotic outage results and observe that the error floor occurs for both systems even at moderate values of $P_{S} / \sigma_{D}^{2}$ due to the residual LI at the relay. The variable-gain AF-FD relaying has much higher error floor and reaches to the error floor at lower $P_{S} / \sigma_{D}^{2}$ values compared to the fixed-gain AF-FD relaying.

In Fig. 5, the consistency of the lower-bound analyses for both the fixed-gain and variable-gain AF-FD relaying are demonstrated where the system parameters are chosen as $2 P_{S}=3 P_{R}, \Omega_{S R}=1, \Omega_{R D}=2, \Omega_{L I}=0.025$, $m_{S R}=4, m_{R D}=2.5, m_{L I}=1$ and $\gamma_{t h}=4$. It is observed that the exact outage probability of the fixed-gain AFFD systems is tightly lower-bounded by the expression given in (32). For instance, there is only $0.38 \mathrm{~dB}$ difference between the lower-bound and the exact outage probability curves when the outage probability equals to $10^{-4}$. In addition, the lower-bound overlaps with the exact outage probability at the error floor. For the variable-gain AFFD relaying, the lower-bound results obtained from (37) are close to the exact outage performance, e.g., the gap between the lower-bound and exact outage probability curves is approximately $1.4 \mathrm{~dB}$ at $2 \times 10^{-2}$. As in the fixedgain AF-FD relaying, the lower-bound reaches to the exact performance at the error floor.

Figure 6 indicates the outage probability versus $\Omega_{L I}$ for $P_{S} / \sigma_{D}^{2}$ values of $10 \mathrm{~dB}, 20 \mathrm{~dB}$ and $30 \mathrm{~dB}$ with $m_{S R}=3$, $m_{R D}=m_{L I}=2.5$ and $\gamma_{t h}=2$. It is seen that the fixedgain AF-FD relaying achieves better outage performance as long as $\Omega_{L I}$ and $P_{S} / \sigma_{D}^{2}$ increases. On the other hand, the variable gain AF-FD relaying has lower outage probability for the small values of $\Omega_{L I}$, e.g., when $\Omega_{L I}$ is smaller than approximately $-2,-16$ and $-24 \mathrm{~dB}$ for $P_{S} / \sigma_{D}^{2}$ equals to $10 \mathrm{~dB}, 20 \mathrm{~dB}$ and $30 \mathrm{~dB}$, respectively. Moreover, it is observed that the outage probability values for both the fixed-gain and variable-gain AF-FD systems are higher than $5 \times 10^{-1}$, even for the high SNR values, e.g., $P_{S} / \sigma_{D}^{2}=$ $30 \mathrm{~dB}$, when $\Omega_{L I}$ is set to $\Omega_{S R}=\Omega_{R D}=1$. This example clearly presents the importance of the LI cancellation in the FD transmission.

In Fig. 7, by using the consistency of asymptotic outage probability expressions given in (35) and (40) with the error floors for the fixed-gain and variable-gain AF-FD systems, respectively, the error floors are represented versus $P_{S} / P_{R}$ for $\Omega_{S R}=1.2, \Omega_{R D}=0.8, \Omega_{L I}=\{0.02,0.06,0.1\}$, $m_{S R}=2, m_{R D}=1.4, m_{L I}=1.9$ and $\gamma_{t h}=5$. We observe that the error floors decrease as long as the $P_{S} / P_{R}$ ratio increases for both systems. Actually, this is a predictable outcome because both the end-to-end SINR expressions given in (33) and (38) for $P_{S}, P_{R} \rightarrow$ $\infty$ are directly proportional to $P_{S}$ and inversely proportional to $P_{R}$. Similar to the previous observations, it is also shown that the fixed-gain AF-FD relaying systems has considerably lower error floors. Also, the error floor for the fixed-gain AF-FD relays decreases more sharply in comparison with the variable-gain AF-FD relays, when $P_{S} / P_{R}$ increases.

\section{Conclusions}

New exact outage probability expressions for the fixedgain and variable-gain AF-FD relaying systems were presented over non-identical Nakagami- $m$ fading channels. The outage probabilities were obtained from CDF of the end-to-end SINR at the destination and verified through Monte-Carlo simulations. Our results show that the fixedgain AF-FD relaying provides much better outage performance than the variable-gain AF-FD relaying when the power of the residual LI and SNR increase. We have also presented tight lower-bound and asymptotic outage performance results for both fixed-gain and variable-gain AF-FD relaying. It was observed that the fixed-gain AFFD relaying has much lower error floor with respect to the variable-gain AF-FD relaying. We have shown that $m_{L I}$ and $m_{S R}$ are dominant $m$ parameters on the asymptotic outage performance of the fixed-gain and variable-gain AF-FD relaying, respectively.

\section{Acknowledgements}

The authors would like to thank the editor and anonymous reviewers for their constructive comments which helped to improve the technical quality of this paper.

\section{Authors' contributions}

We, the authors of this paper, declare that all authors have materially participated in the research and the article preparation equally and have contributed equally to this paper. All authors read and approved the final manuscript.

\section{Competing interests}

The authors declare that they have no competing interests.

\section{Publisher's Note}

Springer Nature remains neutral with regard to jurisdictional claims in published maps and institutional affiliations. 


\section{Author details}

${ }^{1}$ Electronics and Communication Engineering Department, Istanbul Technical University, Istanbul, Turkey. ${ }^{2}$ School of Electrical Engineering and Computer Science, University of Ottawa, Ottawa, Canada.

Received: 30 June 2016 Accepted: 18 May 2017

Published online: 19 June 2017

\section{References}

1. D Kim, H Lee, D Hong, A survey of in-band full-duplex transmission: from the perspective of PHY and MAC layers. IEEE Commun. Surv. Tuts. 17(4), 2017-2046 (2015)

2. S Hong, J Brand, JI Choi, M Jain, J Mehlman, S Katti, P Levis, Applications of self-interference cancellation in $5 \mathrm{G}$ and beyond. IEEE Commun. Mag. 52(2), 114-121 (2014)

3. TRiihonen, S Werner, R Wichman, Optimized gain control for single-frequency relaying with loop interference. IEEE Trans. Wireless Commun. 8(6), 2801-2806 (2009)

4. M Duarte, C Dick, A Sabharwal, Experiment-driven characterization of full-duplex wireless systems. IEEE Trans. Wireless Commun. 11(12), 4296-4307 (2012)

5. E Everett, A Sahai, A Sabharwal, Passive self-interference suppression for full-duplex infrastructure nodes. IEEE Trans. Wireless Commun. 13(2), 680-694 (2014)

6. A Sabharwal, P Schniter, D Guo, DW Bliss, S Rangarajan, R Wichman, In-band full-duplex wireless: challenges and opportunities. IEEE J. Sel. Areas Commun. 32(9), 1637-1652 (2014)

7. JN Laneman, DNC Tse, GW Wornell, Cooperative diversity in wireless networks: efficient protocols and outage behavior. IEEE Trans. Inform. Theory. 50(12), 3062-3080 (2004)

8. GK Karagiannidis, Performance bounds of multihop wireless communications with blind relays over generalized fading channels. IEEE Trans. Wireless Commun. 5(3), 498-503 (2006)

9. MO Hasna, MS Alouini, End-to-end performance of transmission systems with relays over Rayleigh-fading channels. IEEE Trans. Wireless Commun. 2(6), 1126-1131 (2003)

10. G Liu, FR Yu, H Ji, VCM Leung, X Li, In-band full-duplex relaying: a survey, research issues and challenges. IEEE Commun. Surveys Tuts. 17(2), 500-524 (2015)

11. L Rodriguez, NH Tran, T Le-Ngoc, Optimal power allocation and capacity of full-duplex AF relaying under residual self-interference. IEEE Wireless Commun. Lett. 3(2), 233-236 (2014)

12. DPM Osorio, EEB Olivo, H Alves, JCSS Filho, M Latva-aho, Exploiting the direct link in full-duplex amplify-and-forward relaying networks. IEEE Signal Process. Lett. 22(10), 1766-1770 (2015)

13. Q Wang, Y Dong, $X X u, X$ Tao, Outage probability of full-duplex AF relaying with processing delay and residual self-interference. IEEE Commun. Lett. 19(5), 783-786 (2015)

14. TP Do, TVT Le, Power allocation and performance comparison of full duplex dual hop relaying protocols. IEEE Commun. Lett. 19(5), 791-794 (2015)

15. NH Tran, LJ Rodríguez, T Le-Ngoc, Optimal power control and error performance for full-duplex dual-hop AF relaying under residual self-interference. IEEE Commun. Lett. 19(2), 291-294 (2015)

16. L J Rodríguez, NH Tran, T Le-Ngoc, Performance of full-duplex AF relaying in the presence of residual self-interference. IEEE J. Sel. Areas Commun. 32(9), 1752-1764 (2014)

17. JS Han, JS Baek, S Jeon, JS Seo, Cooperative networks with amplify-and-forward multiple-full-duplex relays. IEEE Trans. Wireless Commun. 13(4), 2137-2149 (2014)

18. I Krikidis, HA Suraweera, PJ Smith, C Yuen, Full-duplex relay selection for amplify-and-forward cooperative networks. IEEE Trans. Wireless Commun. 11(12), 4381-4393 (2012)

19. K Yang, H Cui, L Song, Y Li, Efficient full-duplex relaying with joint antenna-relay selection and self-interference suppression. IEEE Trans. Wireless Commun. 14(7), 3991-4005 (2015)

20. Z Shi, S Ma, F Hou, KW Tam, in Proc. IEEE GLOBECOM 2015. Analysis on full duplex amplify-and-forward relay networks under Nakagami fading channels (IEEE, San Diego, 2015), pp. 1-6
21. i Altunbas, Koç, A Yongaçoglu, in Proc. 2016 Wireless Days (WD). Outage probability of full-duplex fixed-gain AF relaying in Rayleigh fading channels (IEEE, Toulouse, 2016), pp. 1-6

22. IS Gradshteyn, IM Ryzhik, Table of Integrals, Series, and Products. (Academic Press, New York, 2007)

23. Y Xu, Y Wang, C Wang, KXu, in Proc. Int. Conf. Wireless Commun. and Signal Process. (WCSP). On the performance of full-duplex decode-and-forward relaying over Nakagami-m fading channels (IEEE, Hefei, 2014), pp. 1-6

24. IS Ansari, S Al-Ahmadi, F Yilmaz, MS Alouini, H Yanikomeroglu, A new formula for the ber of binary modulations with dual-branch selection over generalized-K composite fading channels. IEEE Trans. Commun. 59(10), 2654-2658 (2011)

\section{Submit your manuscript to a SpringerOpen ${ }^{\circ}$ journal and benefit from:}

- Convenient online submission

- Rigorous peer review

- Open access: articles freely available online

- High visibility within the field

- Retaining the copyright to your article

Submit your next manuscript at $\$$ springeropen.com 